\title{
Design and analysis of magnetic matrix screw based on green manufacturing concept
}

\author{
Lilin $^{1}$, Wangbo ${ }^{1}$, Quanxiaofeng ${ }^{1}$ \\ ${ }^{1}$ Beijing Institute of Technology, ZH. Zhu Hai, China
}

\begin{abstract}
Lead screws are the most commonly used transmission components in tool machinery and precision machinery, and are widely used in various industrial equipment and precision instruments. However, the screw tooth profile squeezes and friction causes the gear to break, tooth surface wear, tooth surface pitting and other damage, which reduces the service life of the screw; the use of a large amount of lubricants can not ignore the environmental impact. The design and research of this subject are based on the traditional ball screw mechanism, and based on the magnetic field force, the force transmission and state transformation can be realized without contact. It has significant advantages in terms of improving product service life and reducing pollution caused by lubricating fluid, which is in line with the trend of green and sustainable development. This paper analyzes the principle of non-contact magnetic matrix structure and transmission principle, and conducts finite element analysis on the parameters of screw thrust and torque. Finally, a noncontact screw experimental platform is designed to verify the screw thrust.
\end{abstract}

\section{Introduction}

The traditional ball screw is a transmission device that converts rotary motion to linear motion or linear motion to rotary motion through mechanical connection. In the process of torque and force conversion, frictional resistance is unavoidable, because mechanical connection always exists ${ }^{[1-3]}$. The friction resistance has always existed, and the transmission efficiency has also been affected. At the same time, there are various hazards that damage the overall structure of the machine due to the mechanical connection, such as wear, vibration, noise and other shortcomings, there are unavoidable mechanical transmission parts ${ }^{[5-6]}$. In order to reduce these unavoidable shortcomings, various protections will be installed or added. The device or measure not only affects the performance of the device, but also increases the cost for subsequent maintenance.

The magnet can realize the force transmission and state transformation through the magnetic field force when the object is not in contact. At the same time, it can also avoid the phenomenon of wear, noise, vibration, etc. due to mechanical connection, and can also break through the speed limit. Under magnetic coupling, the vibration of one part will not increase the wear or damage the other part, because the parts are in a non-contact state, there is no frictional resistance, and the parts will not be worn. At the same time, it also realizes ultra-quiet operation and realizes $100 \%$ transmission efficiency ${ }^{[7]}$. The working method of lubrication meets the requirements of green production.

\section{Non-contact screw structure design}

\subsection{Permanent magnet screw structure design}

This paper uses the permanent magnet screw as the research object. The essence of either traditional screw or magnetic matrix screw is the conversion between linear motion and rotary motion. In this study, the thread parts of the permanent magnet screw and nut are replaced by permanent magnets attached to the surface of the cylindrical sleeve ${ }^{[8]}$. Flexible magnets were used instead of the screw, and annular radial magnets were used instead of the sleeve. The magnetic field was arranged through the Halbach array. In this way, the whole screw and sleeve form a closed magnetic field in the radial direction. Its structure is shown in Figure 1 below. The overall structural design is shown in Figure 2. 


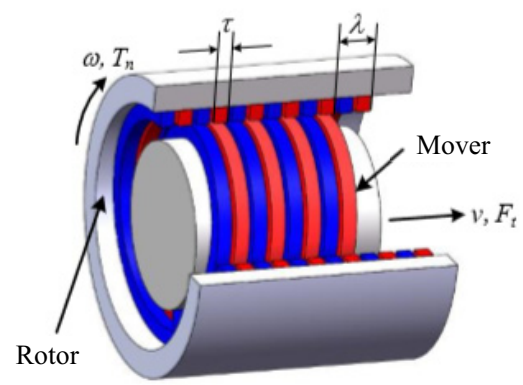

Fig.1 Structure diagram of permanent magnet magnetic lead screw

In the equilibrium state, the magnetic track of the nut and the lead screw in the same direction is opposite to each other. In this state, there is only suction force between the lead screw and the nut. The suction force is when the arrow points to the lead screw, and the repulsion force is when the arrow points to the nut. The nut is suspended, stationary. When screw rotation, track moves forward, at this point, we came to the position of the figure 3, between screw and nut the same magnetic track dislocation, a portion of the magnetic instead of facing, relative to the nut, at this point, the first part of received screw suction, the middle is the repulsion to the left, in the second part is to the left of the suction, the attraction and repulsion, acting in the nut offset each other, the radial direction in the axial direction, is to the left, so the nut was thrust to the left, if the thrust is greater than the load on the nut, nut move left, so, it has realized the rotation movement into linear motion.

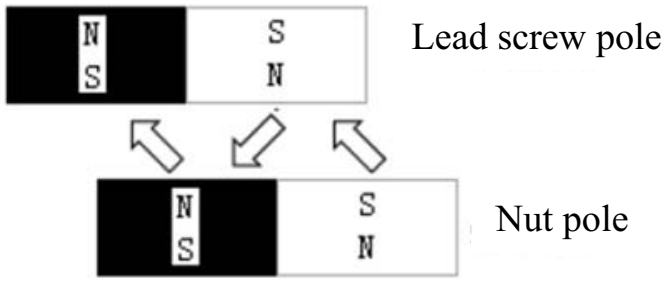

Fig. 3 Transmission Schematic Diagram

\subsection{Analysis of transmission ratio of non-contact screw}

On the whole, the permanent magnet lead screw completes the transfer of mechanical energy, and the form of energy does not change. However, from the point of view of the intermediate process, this mechanical energy transfer is not completed by the mechanical transfer between the rotor and rotor, but depends on the magnetic coupling force between the outer rotor and rotor. The magnetic coupling force is generated by the interaction between the rotor and rotor magnetic fields, so the mechanical energy transfer between the rotor and rotor is mediated by the air gap magnetic field. If the loss in the transfer process is ignored, then:

$$
T_{n} \cdot \omega=F_{t} \cdot v
$$

$T_{n}$ - Torque on the rotor $(\mathrm{Nm})$;

$\omega-$ Rotor angular velocity $(\mathrm{rad} / \mathrm{s})$;

$F_{t}-$-Axial thrust on the mover(N);

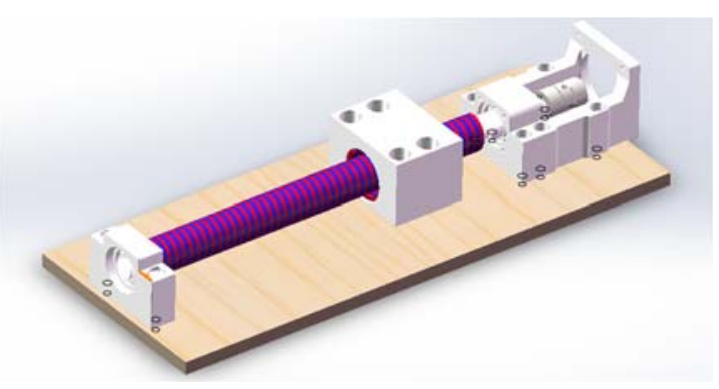

Fig. 2 Structure diagram of lead screw

$v$ - Linear velocity of mover( $\mathrm{m} / \mathrm{s})$.

One rotation of the rotor in the lead screw drives the outer rotor to move in the axial direction. The magnitude of the displacement is equal to its lead, expressed as:

$\lambda-$ Lead of the screw $(\mathrm{m})$

$$
\lambda=2 p \tau
$$

$p-$ Number of poles of permanent magnet;

$\tau$-Permanent magnet pole pitch(m).

Define the ratio of the rotor angular velocity of the magnetic screw to the linear velocity of the outer rotor as the transmission ratio, then:

It follows that:

$$
G=\frac{\omega}{v}=\frac{2 \pi}{\lambda}
$$

$$
\frac{F_{t}}{T_{n}}=\frac{\omega}{v}=G=\frac{2 \pi}{\lambda}
$$

Through analysis, it can be seen that under a certain transmission ratio, no matter how many pole logarithms are, the thrust and torque are positively correlated with the diameter of the lead screw. The larger the diameter of the lead screw, the greater the thrust and torque. However, the magnetic pole logarithm affects the slope of the curve. The slope of the magnetic pole logarithm of 1 is high, and the growth rate of the positive correlation is faster. The result shows that the diameter of the lead screw has a great influence on the thrust and torque. When designing the non-contact ball screw, the desired thrust and torque can be obtained by changing the diameter of the lead screw in consideration of the actual situation.

\section{Analysis of the finite element model of the screw}

The magnetic field distribution inside the screw, especially the air gap magnetic field, determines the working torque, pulsating torque, and transmission efficiency of the magnetic screw. Unlike permanent magnet motors, the magnetic screw has no spatial periodicity, so when modeling, a complete model within a $360^{\circ}$ range is established. In addition, in order to ensure the accuracy of the calculation, the magnetic screw has an air gap and uses a very fine network to divide the low permeability area.

When studying the magnetic screw, because different materials have different magnetic field characteristics, it is necessary to divide the internal field into different solution domains according to different material properties. Compared with permanent magnet motors, the internal 
field division of the magnetic screw is much more complicated, including the inner and outer permanent magnet pole areas, the air gap area, the adjustable magnet core area, and the magnetic ring air slot area. Select radial magnetic flux rotating magnetic screw as the analysis object. The permanent magnet material is neodymium iron boron, and uses radial magnetization. The rotor yoke and outer rotor shaft are made of steel, which provides a path for magnetic field closure. The model uses a four-node tetrahedron as the 3D mesh division unit.

\subsection{Thrust-displacement analysis}

The thrust-displacement characteristic of the magnetic screw reflects the axial thrust and the relative position change of the rotor, and is an important index to measure the control characteristics of the magnetic screw. When the permanent magnet type magnetic screw is working, the rotor rotates around the axis and carries the outer rotor to move linearly in the axial direction. The relative position of the outer rotor and the rotor is fixed. In order to calculate the thrust-displacement characteristics, in the finite element method model, the rotor is fixed, the outer rotor moves linearly along the axial direction, and the movement range of the outer rotor is always within the magnetic pole range of the rotor to ensure the magnetic field coupling area of the outer rotor and the rotor Without changing, the model is calculated and solved, and the thrust-displacement curve of the permanent magnet magnetic screw is obtained.

Using the three-dimensional finite element model to calculate the thrust-displacement characteristics can also obtain the torque-displacement characteristics, as shown in Figure 4.

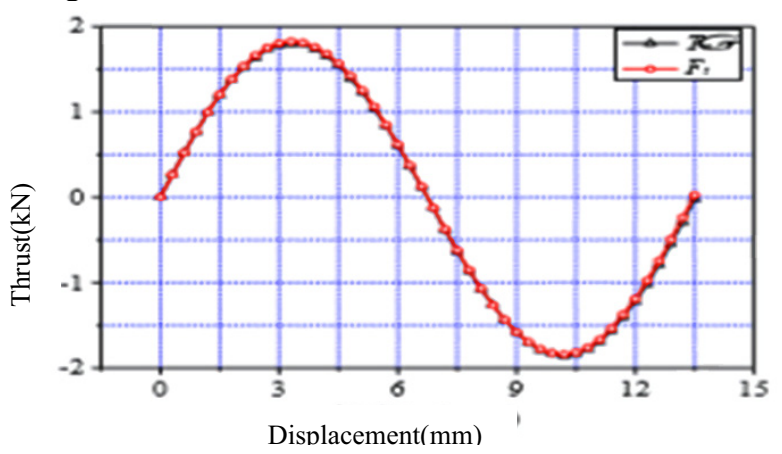

Fig.4 Permanent magnet magnetic screw thrust-displacement curve

As shown from the above figure, the thrust displacement curve of the permanent magnet magnetic screw is basically sinusoidal, so the thrust of the permanent magnet magnetic screw can be approximately expressed as:

$$
F_{t}(z)=F_{\text {tmax }} \cdot \sin \left[\omega\left(z+z^{\prime}\right)\right]
$$

By using three-dimensional finite element model to calculate thrust-displacement characteristics, torquedisplacement characteristics can also be obtained. According to Equation (2), the torque-displacement curve can also be obtained indirectly through the torquedisplacement curve, and the two curves almost coincide, verifying the correctness of the analysis on the operation mechanism of the permanent magnet screw. The peak thrust calculated by this model is $150 \mathrm{~N}$, and the thrust density reaches $5 \mathrm{kN} / \mathrm{m}^{3}$. If the magnetic screw and rotating motor are combined to form a linear motion system, the thrust density is much greater than that of the traditional linear motor.

\section{Comparative analysis of experimental data}

The magnetic lead screw test platform is set up as shown in Figure 6 below. The nut magnetic ring is connected with the translator to make the translator move in a straight line forward, and the other side of the translator is connected with a pressure sensor. The thrust of the translator and the displacement along the axis can be measured through the pressure generated by the spring compression. Under the condition that the screw diameter is $2 \mathrm{~cm}$, the maximum thrust is $171 \mathrm{~N}$, the maximum torque is $0.87 \mathrm{~N} \cdot \mathrm{m}$, the transmission ratio $\mathrm{G}=\mathrm{F} / \mathrm{T}=196.25$, the lead $\lambda$ is $3.2 \mathrm{~cm}$, the series $\mathrm{P}$ is 1 , the pole distance $\tau=\lambda / 2 \mathrm{P}=1.6 \mathrm{~cm}$, the linear velocity of the translator $\mathrm{V}=0.02 \mathrm{~m} / \mathrm{s}$, and the angular velocity of the rotor $3.925 \mathrm{rad} / \mathrm{s}$. Through the experiment, the thrust displacement curve is approximately sinusoidal, as shown in the figure below:

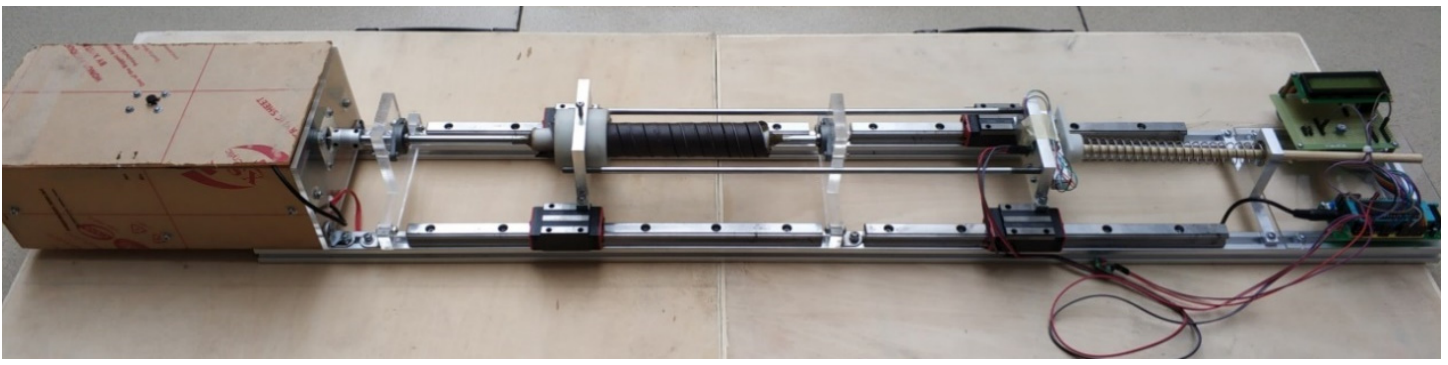

Fig. 5 Magnetic screw test platform 


\section{Conclusions}

After the successful assembly and debugging of the whole experimental platform, we basically achieved some basic functions and characteristics of the target permanent magnet magnetic matrix screw. And the feasibility of the permanent magnet magnetic matrix ball screw is verified through experiments, and through a series of calculations, the influence factors of its thrust output are obtained. The experiments of screw diameter, pole logarithm, pole pitch and air gap length were carried out on this experimental platform, and the effects of each parameter on thrust and torque were verified. The simulation model is verified and modified according to the experimental data, and the accuracy of simulation is improved.

The simulation model is used to analyze the parameters of the lead screw for the Halback magnetizing mode, and it is verified that this magnetizing mode is more efficient than the double-helicity type. The subsequent experiments will be carried out to further improve the Halback magnetic matrix lead screw experimental platform and verify its feasibility.

\section{Acknowledgments}

This article is one of the phased achievements of the young creative talents project in Guangdong Province, "Theoretical Analysis, Structural Design and Optimization of Small Halbach Slotless Wave Linear Generator".

\section{References}

1. Pakdelian S, Toliyat H. Design aspects of the trans- rotary magnetic gear[C]//ECON 2012-38th Annual Conference on IEEE Industrial Electronics Society. IEEE, 2012: 1720-1725.

2. Pakdelian S, Frank N W, Toliyat H. Magnetic Design Aspects of the Trans-Rotary Magnetic Gear[J]. Energy Conversion, IEEE Transactions on, 2015, 30(1): 41-50.

3. Pakdelian S, Toliyat H A. Trans-rotary magnetic gear for wave energy applicaion[C]//Power and Energy Society General Meeting, 2012 IEEE. IEEE, 2012: 1-4.

4. Benjamin A. Magnetic screw: U.S. Patent 1,562,730[P]. 1925-11-24.

5. Faus H T. Magnetic transmission: U.S. Patent 2,371,511[P]. 1945-3-13.

6. Jiabin W, Atallah K, Weiya W. Analysis of a Magnetic Screw for High Force Density Linear Electromagnetic Actuators[J]. Magnetics, IEEE Transactions on, 2011, 47(10): 4477-4480.

7. Smith W, Fukamachi K, Weber S, et al. The Cleveland Clinic/Foster Miller MagScrew pulsatile blood pump program $[\mathrm{C}] / /$ Engineering in Medicine and Biology, 2002. 24th Annual Conference and the Annual Fall Meeting of the Biomedical Engineering Society EMBS/BMES Conference, 2002. Proceedings of the Second Joint. IEEE, 2002, 2： 1561-1562.

8. Schenk S, Weber S, Luangphakdy V, et al. MagScrew total artificial heart in vivo performance above 200 beats per minute[J]. The Annals of thoracic surgery, 2005, 79(4): 1378-1383. 